\title{
Asentimientos informados en jóvenes de 12 a 17 años con patología oncológica: un dilema ético ${ }^{1}$
}

\author{
Claudia López Donoso ${ }^{2}$
}

\section{RESUMEN}

El estudio, cuyos resultados presentamos en este artículo, buscó ahondar en el tema de los Asentimientos Informados como una práctica no común en las atenciones de salud en pediatría. El proceso del asentimiento representa un momento de incertidumbre y de temor. Con esta investigación se buscó conocer la manera en que se reconoce el Asentimiento Informado, los valores que surgen en este proceso y la aplicabilidad que tienen estos instrumentos en salud. Para ello se realizó un análisis de contenido de la información obtenida con tres actores claves de este proceso: los/las jóvenes que poseen una patología oncológica, los cuidadores y el equipo de salud de la Unidad de Oncología del Hospital Luis Calvo Mackenna, a partir de matriz de discernimiento ético (Ver - Juzgar - Actuar).

Palabras clave: Asentimiento informado, autonomía, toma de decisiones

\section{Informed consent in 12 to 17 year-old young people suffering an oncological pathology: an ethical dilemma}

\section{ABSTRACT}

This study seeks to deepen into the issue of Informed Consent, as an uncommon practice in pediatric healthcare. The consent process represents moments of uncertainty and fear. This research is aimed at knowing the way in which the Informed Consent is recognized, values that arise in this process and the applicability of the informed consent in healthcare. For that end, it carried out a content analysis in three key actors in this process: young people suffering an oncological pathology, their caregiving mothers and healthcare team of the Oncology Unit in Luis Calvo Mackenna Hospital, based on the matrix of ethical discernment (See - Judge - Act)

Keywords: Informed consent, autonomy, decision making

1 Articulo recibido 28/11/2018. Artículo aprobado 10/12/2018.

2 Chilena, Trabajadora Social, HOSPITAL LUIS CALVO MACKENNA, E-mail: manclau@gmail.com, clopez@calvomackenna.cl 


\title{
Assentimentos informados em jovens de 12 a 17 anos com patologia oncológica: um dilema ético
}

\begin{abstract}
RESUMO
O estudo cujos resultados apresentamos neste artigo, buscou aprofundar no tema dos Assentimentos Informados, como uma prática não comum nas atenções de saúde em pediatria. $\mathrm{O}$ processo do assentimento representa um momento de incerteza e de temor. Com esta pesquisa busca-se conhecer a maneira em que se reconhece o Assentimento Informado, os valores que surgem neste processo e a aplicabilidade que têm estes instrumentos em saúde. Para aquilo foi realizada uma análise de conteúdo da informação obtida com três atores-chave deste processo: os/as jovens que possuem uma patologia oncológica, os cuidadores e a equipe de saúde da Unidade de Oncologia do Hospital Luis Calvo Mackenna, a partir de matriz de discernimento ético (Ver - Julgar- Agir)
\end{abstract}

Palavras-chave: assentimento informado, autonomia, toma de decisões

\section{Introducción}

En ocasiones se cuenta con la posibilidad de acompañar a jóvenes durante el proceso de enfermedad y, en el final de éste, es posible conectarse con la racionalidad y la emocionalidad que se encuentra presente en esta situación. Así ocurrió durante el tratamiento de una joven de 14 años con prolongado tratamiento de leucemia y luego de un trasplante de médula ósea, proveniente de un sector rural de la VI región de Chile.

La joven se encontraba extenuada por este tratamiento médico, en el que había soportado una hospitalización sin alta de 10 meses, y sentía que su cuerpo y sus fuerzas ya no daban para seguir esta guerra contra el cáncer. Veía que sus padres, que habían abandonado todo, su casa y sus otros hijos, por estar presentes siempre acompañando a su hija, se estaban viendo destruidos en este largo tiempo. Su reacción era fuerte: "no quiere seguir con este tratamiento", "quiero volver a mi casa", "ya no puedo más", y lo hacía saber a todos los que se vinculaban a ella por alguna atención de salud.

Hablar del tratamiento de una patología oncológica en un joven, significa una clara pérdida de rutinas habituales que lo desvinculan de la vida cotidiana, como el colegio, las actividades extra pro- 
gramáticas, los amigos, para instalarse en el hospital. Se recibe un tratamiento que es altamente invasivo tanto en términos de dolor como de la pérdida de la privacidad e intimidad. Con una duración de menos de 8 meses en el mejor de los escenarios. Pero, además, un tratamiento que no está exento de complicaciones: los pacientes pierden el pelo, su apariencia física no luce bien, en ocasiones y dependiendo del diagnóstico, existen afectaciones corporales que no curan en su totalidad, como cicatrices y amputaciones.

Por tanto, en este contexto, que además posee una carga emocional importante para los/las jóvenes de los 12 años en adelante, no es posible no escuchar este grito de ayuda, no asumir esta apelación de auxilio. Así fue como los dilemas éticos comenzaron a evidenciarse en cada reunión que se organizaba para establecer el tratamiento médico más adecuado para la joven, que intentaba hacerse oír en estas reuniones clínicas.

Las respuestas que se escuchan a menudo en circunstancias parecidas, cuando los/las jóvenes manifiestan la necesidad de ser informados, oídos y con opción de decidir, son similares a estas: "es un niño aun"; "seguramente se encuentra con depresión", "lo más probable es una manipulación que quiere hacernos"; "bueno, la verdad es que quienes mandan son los padres y no los hijos".

Probablemente en varios casos, posibles de identificar por cada uno de nosotros, estas respuestas son una realidad. Sin embargo, también existe un número creciente de jóvenes que reclaman por su derecho a ser informados y poder decidir sobre su tratamiento, $y$ es necesario definir acciones que permitan respetar este derecho, que no busca otra cosa que el reconocimiento de la dignidad de todas las personas y esto incluye a los jóvenes que tienen entre 12 y 17 años de edad.

En este dilema ético se reúnen las percepciones de una tríada que por momentos se alía entre sí, y en otros se transforma en una coalición contra un tercero. Parece entonces interesante conocer cuáles son las miradas que se cruzan en estos actos médicos. Para ello se definió como objetivo general del estudio comprender 
la valoración ética que subyace a los discursos de los pacientes entre los 12 y 17 años, sus familias y el equipo de salud, acerca de los asentimientos informados y su aplicabilidad en la Unidad de Oncología del Hospital Luis Calvo Mackenna. En tanto los objetivos específicos, atendían al método de discernimiento ético conocido como VER - JUZGAR - ACTUAR. "El discernimiento teológico del ver-juzgar-actuar tiene sus raíces en Mater et Magistra (1961), $\mathrm{N}^{\circ}$ 236; Octogésima Adveniens (1971), $\mathrm{N}^{\circ} 4$." (Mifsud, 2006, p. 27). Es conocido como método pastoral del Movimiento de la Acción Católica (Padre Cardijn), en el que se proponían tres pasos de observación de la realidad, reflexionar sobre ella, para proceder con una práctica consecuente. Este método fue asumido por la Iglesia en el campo de lo social (Mifsud, 2006, p. 28). Con ello se construye una matriz de análisis ético, propuesta por Lizana (2010).

Para el componente VER se definió como objetivo describir cómo es reconocido el proceso de asentimiento informado por los distintos actores que participan del tratamiento de una patología oncológica en el Hospital Luís Calvo Mackenna. El segundo objetivo específico, que correspondía a JUZGAR, indagaba en reconocer elementos éticos respecto del asentimiento informado y su aplicabilidad que se develan en los contenidos expresados por los distintos actores del proceso. En el tercer objetivo específico se buscaba dar cuenta de las propuestas de aplicabilidad del asentimiento informado que realizan los distintos actores, respondiendo así al tercer componente del ACTUAR.

Este estudio se realizó, específicamente, en la Unidad Oncológica del hospital público Luis Calvo Mackenna, centro de salud perteneciente al Programa Nacional de Oncología Pediátrica (PINDA). A nivel nacional, debutan al año cerca de 500 casos, de los cuales 120 niños y niñas son atendidos por la unidad de oncología de este hospital, ejecutándose cerca de 300 quimioterapias al mes. Dicha unidad ha sido definida como referencia nacional para los diagnósticos de osteosarcoma (cáncer en los huesos) y es el único centro de salud público y pediátrico en donde se realizan los tras- 
plantes de médula ósea, esperando cumplir con un promedio de 40 trasplantes de médula ósea al año (esta cifra es ajustada anualmente).

\section{Conceptos que nos acompañan}

Para el desarrollo de este estudio, se definieron un conjunto de conceptos de referencia que facilitaran el análisis de la complejidad del proceso y la posterior interpretación de los contenidos identificados. En la realidad hospitalaria del país es posible observar cómo la hegemonía céntrica adulto tiene resonancia aun en las atenciones de salud pediátrica. Nos enfrentamos a un medio en que los jóvenes son vistos a partir de 4 características definidas por Duarte (2000). La primera de ellas dice relación con una concepción mecánica y lineal del ciclo de vida, que asciende hasta la adultez y que, en forma descendente, llega hasta la vejez. Una segunda característica corresponde al universalismo, que consideraría a todos los/las jóvenes como iguales, y por lo tanto vivenciarían sus procesos de vida de igual forma, lo que produce la invisibilización de las diferencias entre jóvenes.

La tercera característica da cuenta del esencialismo asociado a la idealización de la juventud, que se asienta en el vigor y la fuerza de la edad y el espíritu trasformador del mundo. Por último, la cuarta característica corresponde a la estigmatización, que no es otra cosa que sentir que los/las jóvenes son un problema para la sociedad (Duarte, 2000, pp. 59-77).

Tradicionalmente en temáticas del desarrollo moral se reconocen autores como Dewey, Piaget y Kohlberg, cuyos planteamientos se aproximarían bastante a la posición naturalizada que denuncia Duarte de lo que es "la" juventud. No obstante ello, representan tendencias clásicas necesarias de considerar en este debate, para apoyarlas o bien para rebatirlas. Cada uno de estos autores fue agregando elementos a lo planteado por el anterior, que se traducen en clasificaciones de los niños/jóvenes según su grado de madurez bajo criterios que los homogenizan. 
Un cambio se produce en esta tendencia y tiene que ver con el pensamiento de Carol Gilligan, que si bien cumple con algunas de las características anteriores, suma un nuevo elemento que nos parece importante.

Estos autores habían realizado sus trabajos descartando a las mujeres en un principio y luego concluyen que poseen menos desarrollo moral que los hombres. Guilligan realiza su trabajo en mujeres y confirma que mujeres y hombres tenemos diferencias en el desarrollo moral, pero que ambos son valiosos, y plantea una nueva mirada que contiene la ética del cuidado, vinculada a la beneficencia y a la responsabilidad social, poniendo énfasis en el respeto por la diversidad y en la satisfacción del otro (Rueda, 2007). Así también, reconoce las historias particulares del cuidado y valora el bienestar de aquellos que pudieron verse afectados por decisiones morales con consecuencias para la vida.

Estos elementos recién tratados cobran relevancia en este estudio, dado que son los cuerpos teóricos que validan la autonomía y el tiempo en que ésta se consigue. Para hablar de autonomía podemos recordar una primera definición, que indica que sería la "capacidad que tiene la persona humana en tomar sus propias decisiones en relación a su vida, a su salud y a su felicidad" (Gamarra, 2015, p. 3).

Se ha entendido también autonomía como la propia regulación que debiese ocurrir, libre, sin influencias externas que puedan significar control y sin limitaciones personales que impidan hacer una elección (Pellegrino, 1990). Es decir, una persona actúa libremente de acuerdo con lo que ha planificado. Una tercera definición de autonomía hace referencia a la regulación de la conducta mediante normas que el individuo se da a sí mismo. Por lo tanto, "autónomo es todo aquél que decide conscientemente qué reglas son las que van a guiar su comportamiento" (Modelo de desarrollo económico de Navarra, s/f).

Sin embargo, a estas definiciones, que son un buen ejemplo de lo que se considera autonomía, necesariamente se le deben asociar 
algunos aspectos que se constituyen en salvedades del concepto, no menos relevantes. Efectivamente todas las personas tienen la capacidad para la toma de decisiones en su vida, pero esta facultad no es una condición sine qua non; no es a cualquier edad o en cualquier estado de madurez.

Como la madurez es un proceso que se comienza a considerar en edades que se reconocen como parte de la llamada "adolescencia" (no siendo el concepto que la autora emplea en este estudio), es importante recalcar que, frente al tema de la juventud entre los 12 y 17 años, sus definiciones y características no poseen criterios uniformes y existen teorías que avalan la perspectiva relativista y otras que observan la adolescencia desde una mirada universalista. En general, para introducirse al tema de la madurez, ambas teoría entran en juego en este análisis.

En tal sentido, la literatura de índole psicológica, legal y moral detalla que los niños/niñas y los/las jóvenes, entre los 12 y 17 años no poseen esta autonomía para tomar decisiones en su propia vida, sino que es un proceso que se va alcanzando en el trascurso de los años. Carmen Martínez (2013) indica: "La adolescencia es el proceso psíquico que acompaña al desarrollo puberal, al final del cual la persona es autónoma y se supone madura" (p. 23). Pero una persona que adquiere la autonomía legal no necesariamente consigue la madurez a los 18 años.

Desde una óptica universalista, durante la edad a la que hacemos referencia ocurren cambios de índole físico y psicológico en los/ las jóvenes, que tienen como resultado la adquisición de una identidad propia, para lo que se hace necesario salir de la dependencia familiar.

Este proceso, además, se ve acompañado por el paso a la madurez. Según la misma autora, la madurez sería reconocida como "la capacidad para asumir las convenciones sociales desde una elaboración autónoma de los principios morales" (Martínez, 2013, p. 24). Esto no es otra cosa que el camino que va desde la heteronomía de los niños, en que las normas vienen de fuera y se cumplen sin 
cuestionamiento, hacia una autonomía moral, en las que, según Kohlberg plantea, la persona crea sus propios criterios y toma las decisiones según su propio pensar.

En nuestra sociedad se otorga a los/las jóvenes de entre 12 y 17 años atribuciones ante la vida diferenciadas que suenan contradictorias. Por ejemplo, cuando se habla de la ley de responsabilidad penal juvenil, que sanciona a los/las jóvenes que hayan cometido delito entre los 14 y 18 años, o cuando se plantea la posibilidad del uso de anticoncepción o de la pastilla del día después, sin que para esto sea necesario contar con la autorización de los padres. Estas diferencias en los alcances en las facultades de los derechos de los/ las jóvenes, tienden a generar confusiones en otros procesos de la vida ante los cuales debiesen tomarse decisiones, como es el tratamiento médico ante una enfermedad.

En un artículo elaborado por el Instituto Libertad y Desarrollo, bajo el título "Menores en Chile: ¿Cuándo son capaces o no son capaces?" del año 2012, se indica que "(...) parece evidente la necesidad de que el legislador uniforme los criterios y en la medida que otorgue a los menores de edad un mayor grado de autonomía, restrinja también la esfera de responsabilidades de padres o guardadores" (p. 2).

A continuación se muestra una tabla general de cómo la legislación chilena reconoce las capacidades de niños y niñas.

Tabla 1: Legislación chilena y capacidades de niños y niñas.

\begin{tabular}{|l|l|l|l|}
\hline Infante & Impúber & Menor/adulto & Adulto \\
\hline $\begin{array}{l}\text { Menores de 7 } \\
\text { años }\end{array}$ & $\begin{array}{l}\text { Mujer: 7 a 12 años } \\
\text { Varón: 7 a 14 años }\end{array}$ & $\begin{array}{l}\text { Mujer: 12 a } 18 \text { años } \\
\text { Varón: 14 a } 18 \text { años }\end{array}$ & $\begin{array}{l}\text { Mayores de } 18 \\
\text { años }\end{array}$ \\
\hline $\begin{array}{l}\text { Incapaz Ab- } \\
\text { soluto }\end{array}$ & Incapaz Absoluto & Incapaz Relativo & Plenamente Capaz \\
\hline
\end{tabular}

Fuente: Libertad y Desarrollo (2012), pág. 3. 
Si ha habido un concepto en tensión ha sido obviamente este: ¿hasta dónde los/las jóvenes tienen la posibilidad de ser autónomos? Todo esto se plantea en una forma diametralmente opuesta cuando se evalúa la autonomía para ponerla en práctica en las atenciones de su salud, en enfermedades graves con riesgo de vida y el término o no de un tratamiento doloroso que no tiene un buen pronóstico.

Para hablar de ética y moral debemos partir reconociendo que la ética es una dimensión puramente humana. Como dice Ruth Lizana, "El ser humano es un ser inteligente, en cuanto es capaz de comprender, de crear, de saber; es libre y, por lo tanto, puede elegir y es consciente, es decir, sabe que sabe y que puede elegir, (Lizana, 2010).

La bioética es la instalación de la ética en los actos de salud. Este es un concepto que surge en el contexto de los crímenes ocurridos en la Segunda Guerra Mundial con los nazis. Si fuese posible pensar en algún aspecto positivo de estos hechos, podríamos considerar en la creación del Código de Ética Médica de Núremberg, en el que se reconoce el concepto actual de "consentimiento informado".

El Dr. Van Rensselaer Potter (s.f.) crea una teoría disciplinar de la bioética, que busca "estudiar los problemas morales surgidos al calor del desarrollo científico y que abarca no sólo al hombre sano o enfermo, sino a todos los seres vivos que tienen relación con la mejor calidad de vida" (Potter, citado en Amaro, 1996, p. 3).

Para esto, se definen cuatro principios que se emplean para evaluación de las acciones médicas desde la bioética. Si bien estos principios es posible reconocerlos en forma individual, es necesario mencionar que se encuentran íntimamente relacionados y se detallan a continuación.

- Principio de la Beneficencia: significa hacer el bien en todas las acciones desarrolladas en la atención de salud.

- Principio de la No Maleficencia: Significa no hacer mal. Este principio necesariamente se debe observar nuevamente de acuerdo a los ethos epocales, y por cierto, a los valores morales de un momento histórico dado. 
- Principio de Justicia: se refiere a la justicia redistributiva, entendiendo por esto la distribución de los bienes /recursos que siempre son escasos en un comunidad.

- Principio de la Autonomía: se entiende como "la aceptación del otro como agente moral responsable y libre para tomar decisiones" (Amaro et al., 1996. p. 4). De este principio, se desprenden los consentimientos informados en la atención de salud.

El acto de asentir o consentir, en la actualidad, no solo es una acción de conciencia médica, sino que corresponde a una normativa establecida por el Estado de Chile mediante la Carta de Deberes y Derechos de los Pacientes, finalmente reconocida legalmente en el año 2012 (MINSAL 2012). En el caso de los/las jóvenes, esto no es tan evidente como en los adultos; aún más cuando se considera en uno de los principios de la bioética que habla de "beneficencia y no maleficencia”.

En este principio es el médico quien toma las decisiones, dado que es quien cuenta con el conocimiento técnico para ofrecer la mejor opción para el paciente. En definitiva, lo que se traduce de esta conducta es que implica una participación de los jóvenes algo limitada (Espinosa, Menoret Puchulu, Selios y Witriw, 2009).

Los documentos revisados esbozan que es el facultativo médico quien está llamado a determinar cuándo las capacidades y las habilidades de una persona le permitirían firmar el consentimiento/ asentimiento. En este sentido, se plantea el permanente cuestionamiento respecto de la edad en que un joven pasa a ser considerado como un Niño Maduro, habilitándolo finalmente para la toma de decisiones. Nos enfrentamos a dilemas éticos cuando la opinión vertida por el médico es opuesta a la del joven y la de los padres, y se requiere lidiar con estas diferencias. Como una forma de hacer efectivo el derecho de los jóvenes a expresar su opinión respecto de un tratamiento médico se ha dado cuerpo a los conceptos de consentimiento y asentimiento informado.

El término que primero se acuñó fue el de "consentimiento informado", que tiene implicancias dirigidas a las personas adultas, 
para luego transitar hacia un cambio del término de tal forma que permitiese hacerse cargo de este proceso en las personas jóvenes, así surge el concepto de "asentimiento".

El consentimiento informado, tal como lo ha planteado Laura Rueda (2007), habla de un intercambio de información que debiese fluir entre el paciente/enfermo y quienes le darán la atención de salud. Plantea el término de intercambio como el hilo conductor, por tanto, este proceso necesariamente incluye a alguien que pregunta y otro que responde y luego hay un cambio del rol; es decir, quien respondió se transforma en quien pregunta. No se ha planteado aquí una relación unidireccional (Rueda, 2007). De forma similar lo ha indicado Alina Macías (2006), quien incorpora a esta descripción aspectos interesantes de valorar. Primero, se reconoce al consentimiento y asentimiento como un proceso y, por ende, posee características de gradualidad.

Se indica junto a esto que es un proceso verbal que se desprende de la relación médico/paciente. Para que esta trama comunicacional se realice de esta manera, se hace necesaria una segunda característica, que se vincula con el contacto interpersonal de confianza entre el equipo de salud y el paciente, lo que se reconoce en el ámbito de la salud como la "relación clínica".

Ambas autoras mencionan que el consentimiento se enmarca en un proceso de intercambios en los que se sostiene la relación. Por tanto, no es posible imaginar una firma de este valioso documento, por su envergadura, entre personas extrañas y sin que la acción se desprenda de varios contactos previos entre el médico y el paciente.

Francisco León (2012) formula una definición interesante de esta relación, cuando señala que, para ejercer la función de cuidado de la salud como experto, necesariamente se requiere establecer una alianza con los pacientes. Menciona además que esta relación se trasforma en un diálogo entre agentes morales.

El consentimiento, al incorporar la relación con el médico, debe incluir el requisito improrrogable de la entrega de información. 
Esto es relevante cuando esta información permite tomar una decisión para un tratamiento médico que pudiese tener como consecuencia una mejora en la condición de salud o el empeoramiento de ésta.

Efectivamente, el consentimiento informado debe hacerse cargo del objetivo de informar de manera comprensible y relevante. Informar inadecuadamente conduce directamente a la firma de consentimientos desinformados, que es éticamente incorrecto pero universalmente se ha ido aceptando por los centros hospitalarios (Mecías, 2006).

Un tercer aspecto dentro del proceso de consentimiento se relaciona con la voluntariedad o la libertad para elegir uno u otro tratamiento. Es decir, este no pudiese ser firmado si para esto se empleó la coacción física o emocional, que a su vez genera una auto imposición interna del paciente. El médico cubano Pedro González Fernández expresa: "Hay que tratar de que el proceso de información y consentimiento no se convierta en un acto con un trasfondo "defensivo", en el cual el médico busque "protección" contra una posible demanda o acusación" (González, 2007, p. 2), y tal como lo indica el mismo autor, "Para que ello marche bien, la compasión y la comunicación son elementos esenciales".

En el caso de la comunicación, antes de plasmarse en un documento, "debe estar precedida por una conversación movida por amor oblativo hacia ese ser humano" (González. 2007, p. 3), poniendo a este acto como una acción de humanización de la atención de salud.

De esto es posible indicar que este intercambio de información debe considerar tres elementos fundamentales:

- Revelación de la información, que se refiere al tipo de procedimiento, sus implicancias, consecuencias, sus riesgos y beneficios.

- Capacidad (o competencia), que hace referencia a las habilidades comprensivas de una persona para ser capaz de evaluar riesgos y beneficios. 
- Voluntariedad: se refiere al derecho de un paciente a tomar las decisiones relativas a su tratamiento libre de cualquier tipo de influencia indebida.

Tal como se mencionó, hay un segundo concepto que se ha desprendido del consentimiento y que corresponde al asentimiento.

El concepto de asentimiento hace referencia a la incorporación de los niños, niñas y jóvenes en la toma de decisiones sobre sus tratamientos médicos (Bustamante, 2013). Si bien este término es reconocido por la generalidad de los médicos, su ejecución genera habitualmente un problema ético, dado por el momento en el que se puede considerar o no a un niño/niña/joven con capacidades para la toma de decisiones.

Con el reconocimiento de lo imprescindible que es este acto para los pacientes hoy en día, ningún equipo de salud dudaría de realizar este proceso y existe conciencia de que la información no es del médico, sino del paciente.

Así también lo indica la Ley de Deberes y Derechos que se mencionó, y que manifiesta claramente en su Artículo $8^{\circ}$ : Toda persona tiene derecho a que el prestador institucional le proporcione información suficiente, oportuna, veraz y comprensible, sea en forma visual, verbal o por escrito (Ley 20.584).

Indica, además, otros antecedentes relevantes:

- Toda persona tiene derecho a ser informada, en forma oportuna y comprensible, acerca de su estado de su salud, del posible diagnóstico de su enfermedad, de las alternativas de tratamiento disponibles para su recuperación y de los riesgos que ello pueda representar, así como del pronóstico esperado, y del proceso previsible del postoperatorio cuando procediere.

- Información de acuerdo a la edad y a la condición personal y emocional.

- Cuando la condición de la persona, a juicio de su médico tratante, no le permita recibir la información directamente, será 
dada a su representante legal o, en su defecto, a la persona bajo cuyo cuidado se encuentre.

- Recuperándose de la conciencia y capacidad de comprensión que le impide recibir la información, deberá ser informada de la mejor forma posible.

Una nueva propuesta que se trabaja en el hospital Luis Calvo Mackenna corresponde al Modelo de Hospital Amigable creado por dos enfermeros ingleses, Sue Anderson y Andrew Clarke, que plantea como objetivo reducir el miedo, la angustia y el dolor en el niños, niñas y adolescentes y en su familia, provocados por las atenciones de salud. Este modelo ha tomado el derecho de los niños y los ha traducido a estándares que implican la operacionalización de estos en salud.

Para el derecho a la información, el Modelo de Hospital Amigable definió el estándar 5, que habla de:

Compartir información y mantener a los padres y niños consistente y completamente informados e involucrados en todas las decisiones. Los prestadores de atención médica, tanto organizaciones como funcionarios de salud individuales comparten una responsabilidad de apoyar a los niños y reducir su miedo, ansiedad y sufrimiento, y las de sus familias, al asegurarse de mantenerlos consistente y completamente informados e involucrados en todas las decisiones. (Anderson y Clarke, 2007)

Históricamente, la infancia no siempre ha sido valorada; por tanto, plantearse la idea de informar también a personas de esta edad ha sido un avance significativo en la humanización de la atención de salud. En la historia de la humanidad hemos pasado de etapas de subvaloración de los niños, a una valoración con mayor conciencia en la actualidad.

Se ha transitado entonces desde un modelo tutelar, que considera a los niños como menores objetos de protección a uno en el que se les ha considerado sujetos de derecho. "Estas leyes conciben a los niños y jóvenes como objetos de protección a partir de una defi- 
nición negativa de estos actores sociales" (Bellof, citado en Fuenzalida, 2014, p. 22).

La concepción de los niños como menores los ubicaba en una categoría de incapacidad, siendo ellos solo un receptor de beneficios sociales.

La realidad latinoamericana de crisis económica y el aumento de migraciones favorece el crecimiento poblacional infantil; niños/niñas en abandono y ausentes de las redes de apoyo, nominándolos como "menores en situación irregular". La falta de un Estado con recursos y la ausencia de políticas sociales dirigidas a la infancia hacen que la judicialización sea el instrumento para resolver sus problemas. En 1989, con la ratificación por parte del Estado de Chile de la Convención de los Derechos del Niño, se genera un cambio del modelo tutelar al modelo de protección integral.

En el levantamiento de la información recogida de los informantes clave aparece otro tema: la adherencia a los tratamientos médicos. En la actualidad, la Organización Mundial de la Salud (2003) ha indicado que $50 \%$ es la tasa de adherencia a los tratamientos en enfermedades crónicas en adultos en países desarrollados. A tal nivel es esta consideración que además ha declarado que es un "problema mundial de gran magnitud" (OMS, 2003) y ha mandatado a las naciones y a sus ministerios de salud y a sus símiles a realizar acciones para mejorar estos niveles.

"Adherencia" se entenderá como el proceso a través del cual el paciente lleva a cabo adecuadamente las indicaciones terapéuticas, es decir, cuando cumple las prescripciones recibidas para el manejo de una enfermedad (Ginarte, 2001). La OMS también la ha definido como: "el grado en el que la conducta de un paciente, en relación con la toma de medicación, el seguimiento de una dieta o la modificación de hábitos de vida, se corresponde con las recomendaciones acordadas con el profesional sanitario" (OMS,2003). 
El concepto de "adherencia" incluye dos elementos conformadores que tienen que ver con la aprobación del enfermo de las recomendaciones recibidas desde el médico y una colaboración activa entre el profesional y el paciente en la toma de decisiones que afectan a su propia salud.

La misma Organización Mundial de la Salud ha esgrimido como razones para el mejor cumplimiento de la adherencia, aspectos que tienen que ver con el mismo tratamiento y con el personal de salud. Aquellas que se relacionan con el paciente se refieren a la edad, el entorno cultural y social del paciente, el nivel de educación, así como su personalidad, lo que condiciona el resultado del tratamiento. La presencia de problemas psicológicos, como depresión o ansiedad, y las posibles creencias y percepciones del paciente sobre la medicación son aspectos que afectan la adherencia.

Así también, las explicaciones relacionadas con el tratamiento se vinculan al tipo de fármacos sus efectos y las dificultades para su ingesta. El tipo de procedimiento médico indicado y las sensaciones de desagrado que este puede generar hacen que con mayor facilidad el paciente pierda la motivación para la continuidad del mismo. Costos de las indicaciones (fármacos/alimentos/insumos, etc.) y una falta de monitoreo periódico del paciente, son causales observadas a diario. Mientras más extensos son los tratamientos menos perseverantes son los pacientes.

Las razones de los profesionales del ámbito sanitario se vinculan a la relación médico/paciente, que cada vez cobra mayor relevancia. La falta de tiempo, las dificultades en la comunicación entre ambos, la falta de la entrega de información sobre la enfermedad y el tratamiento, son aspectos que no ayudan a la mantención de la adherencia. Un aspecto importante, corresponde a la confianza que debe existir entre el equipo de salud y el paciente. Los conceptos clave de este estudio se reflejan en el siguiente mapa conceptual. 
Figura 1: mapa conceptual.

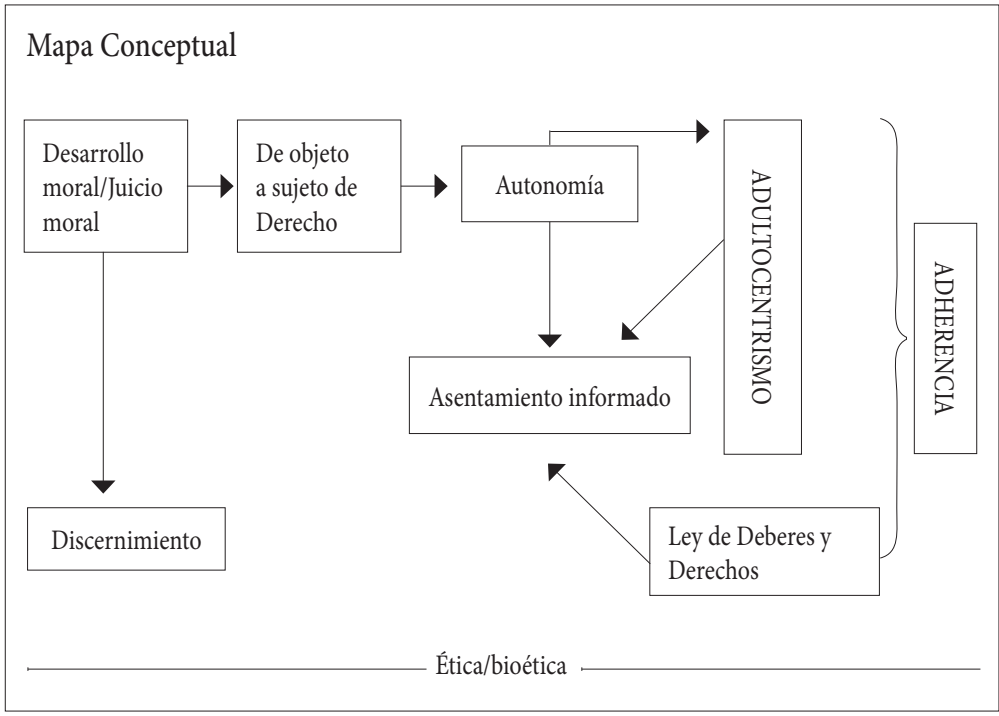

Fuente: Elaboración propia.

\section{Metodología}

En cuanto a la metodología, se trató de un estudio de caso con alcance exploratorio-descriptivo y enfoque cualitativo. Las unidades de estudio estuvieron conformadas por los/las jóvenes de entre 12 y 17 años, familiares (madres) y equipo de salud. Es importante señalar que fueron finalmente entrevistadas las madres, porque aún son ellas las que asumen los cuidados en situaciones de enfermedad.

Para la selección de los pacientes, se definieron criterios tales como:

- Jóvenes entre 12 y 17 años en tratamiento oncológico activo.

- Madres con hijos en tratamiento oncológico activo.

- Equipo de salud, constituido por técnicos y profesionales con contratos vigentes en la unidad de oncología.

Las técnicas de recolección de información definidas fueron:

- Jóvenes: entrevista semiestructurada.

- Madres: entrevistas semiestructurada.

- Equipo de salud: entrevista especializada y a elite 
Asentimientos informados en jóvenes de 12 a 17 años con patología oncológica: un dilema ético /López

\section{Resultados}

Del total de 26 funcionarios estables de la unidad, fueron parte de este estudio 12 de ellos, lo que se distribuyó de la siguiente forma:

Tabla 2: Muestra equipo de salud.

\begin{tabular}{|l|c|c|}
\hline Equipo de salud & $\mathrm{N}^{\circ}$ & Promedio de años oncologia \\
\hline Médicos & 2 & 26 \\
\hline Enfermeras & 5 & 16,6 \\
\hline Enfermera paliativista & 1 & 15 \\
\hline Técnico paramédico & 1 & 4 \\
\hline Psicólogas & 2 & 6 \\
\hline Trabajadora Social & 1 & 2 \\
\hline
\end{tabular}

Fuente: elaboración propia.

Respecto de las cuidadoras, se logró entrevistar a 4 de ellas:

Tabla 3: madres a cargo del cuidado de los hijos.

\begin{tabular}{|l|l|}
\hline Madres & Edad del hijo/hija \\
\hline Madre 1 & Hija, 12 años \\
\hline Madre 2 & Hijo, 15 años \\
\hline Madre 3 & Hija, 12 años \\
\hline Madre 4 & Hijo, 14 años \\
\hline
\end{tabular}

Fuente: elaboración propia

Respecto de los/las jóvenes, la distribución correspondía a:

Tabla 4: jóvenes pacientes oncológicos.

\begin{tabular}{|l|l|}
\hline Jovenes & Edad /Sexo \\
\hline Joven 1 & Mujer 12 años \\
\hline Joven 2 & Hombre 14 años \\
\hline Joven 3 & Hombre 14 años \\
\hline
\end{tabular}

Fuente: elaboración propia.

A continuación se presentan mediante, cuadros/síntesis, las categorías y subcategorías que surgen del análisis de los discursos. 
Estos cuadros no expresan la totalidad de los discursos recogidos, pero sí las categorías centrales, refrendadas con algunos relatos textuales a modo de ejemplo.

Respecto del primer objetivo especifico que era describir cómo es reconocido el proceso de asentimiento informado por los distintos actores que participan del tratamiento de una patología oncológica en el Hospital Luís Calvo Mackenna, los resultados arrojan la siguiente información:

Cuadro 1: Resultado dimensión asentimiento informado como derecho: equipo de salud.

\begin{tabular}{|c|c|c|}
\hline $\begin{array}{l}\text { DIMENSION: } \\
\text { Asentimiento } \\
\text { Informado }\end{array}$ & \multicolumn{2}{|l|}{ EQUIPO DE SALUD } \\
\hline Categorias & Sub Categorias & Relato del entrevistado \\
\hline \multirow[t]{2}{*}{$\begin{array}{l}\text { Asentimiento } \\
\text { Informado como } \\
\text { Derecho }\end{array}$} & Derecho a la información & $\begin{array}{l}\text { "cada uno tiene el derecho de ser informado } \\
\text { de su diagnóstico, da lo mismo la edad, puede } \\
\text { ser explicado de acuerdo a capacidad de } \\
\text { entender de cada persona" ( Cl0) }\end{array}$ \\
\hline & Derecho a la autonomia & $\begin{array}{l}\text { “... ya no son niños. Hoy en día los } \\
\text { adolescentes tienen acceso a mucha } \\
\text { información y es importante darles el espacio } \\
\text { para opinar y poder actuar según sus } \\
\text { preferencias. (C5) }\end{array}$ \\
\hline $\begin{array}{l}\text { Cumplimiento del } \\
\text { tratamiento medico }\end{array}$ & Adherencia & $\begin{array}{l}\text { "... permite sentir algo de control en la difícil } \\
\text { situación que atraviesan y mejora adherencia } \\
\text { y colaboración con el tratamiento"(C7) }\end{array}$ \\
\hline
\end{tabular}

Fuente: elaboración propia.

Cuadro 2: Resultado dimensión asentimiento informado como derecho: Madres cuidadoras.

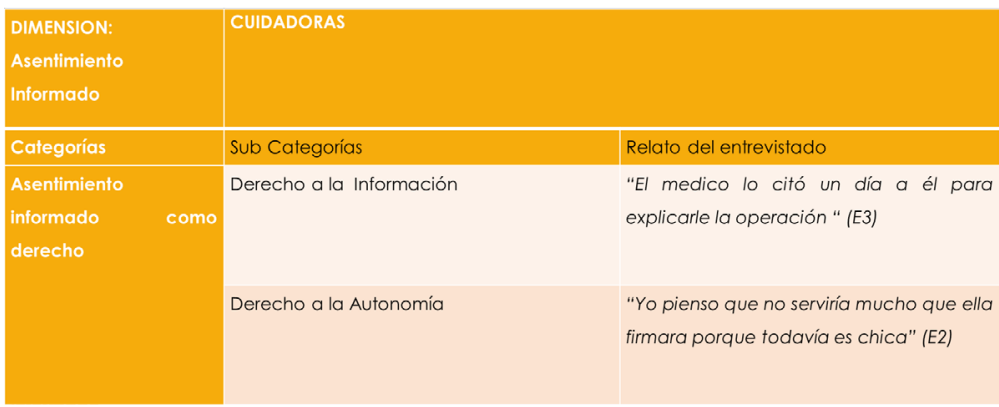

Fuente: elaboración propia. 
Asentimientos informados en jóvenes de 12 a 17 años con patología oncológica: un dilema ético /López

Cuadro 3: Resultado dimensión asentimiento informado como derecho: jóvenes pacientes oncológicos.

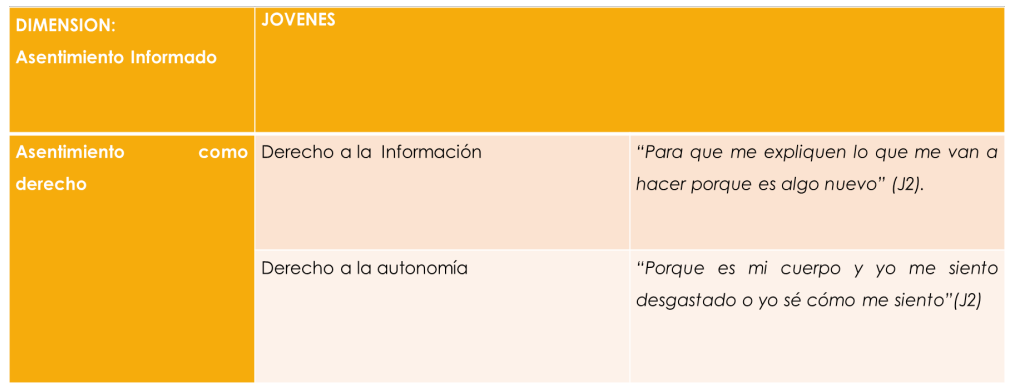

Fuente: elaboración propia.

Respecto del segundo objetivo específico, que era reconocer elementos éticos que se develan, respecto del asentimiento informado y su aplicabilidad, en los discursos de los distintos actores que participan del tratamiento de una patología oncológica en el Hospital Luís Calvo Mackenna, los resultados fueron los siguientes:

Cuadro 4: Dimensión elementos éticos: equipo de salud.

\begin{tabular}{|c|c|c|}
\hline $\begin{array}{l}\text { DIMENSION: } \\
\text { EIICOS }\end{array}$ & EQUIPO DE SALUD & \\
\hline $\begin{array}{l}\text { Calegorías Asociadas al } \\
\text { Objetivo } \mathrm{N}^{\circ} 2\end{array}$ & Sub Categorías & Relato del entrevistado \\
\hline \multirow[t]{3}{*}{ Expresiones valórica } & Valores & $\begin{array}{l}\text { "El principio de respeto por el otro } \\
\text { independiente de su edad: El reconocer } \\
\text { a ese adolescente como personas con } \\
\text { derechos y deberes" ( } C 6 \text { ) }\end{array}$ \\
\hline & Principios Bioéticos & $\begin{array}{l}\text { "Autonomía, beneficencia, no } \\
\text { maleficencia y justicia"( } \mathrm{C} 8 \text { ) }\end{array}$ \\
\hline & Dilemas Éticos & $\begin{array}{l}\text { "El más difícil has sido cuando un } \\
\text { adolescente no quiere un tratamiento y } \\
\text { sus padres si" }(\mathrm{C} 8)\end{array}$ \\
\hline
\end{tabular}

Fuente: elaboración propia. 
Cuadro 6: Dimensión elementos éticos: madres cuidadoras.

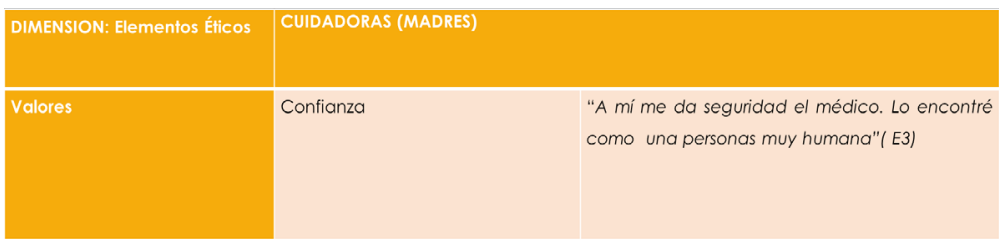

Fuente: elaboración propia.

Cuadro 7: Dimensión elementos éticos: jóvenes pacientes oncológicos.

\begin{tabular}{|c|c|c|}
\hline DIMENSION: & \multicolumn{2}{|l|}{ JOVENES } \\
\hline \multirow[t]{3}{*}{ Alteridad } & Relación con Dios & $\begin{array}{l}\text { "Si, pero yo le pido mucho a Diosito que me haga un } \\
\text { milagro para que yo saiga luego de esto. Yo soy } \\
\text { bailarina y entonces, quiero ir a bailar pero quiero ir } \\
\text { sana. Le pido a Diosito que no me corten mi pie. } \\
\text { Que Dios me haga un milagro y que me saque eso } \\
\text { de ahi y no me corten mi pie. El } 6 \text { de junio me van a } \\
\text { hacer un examen y me van a ver si sigue pegado el } \\
\text { hueso, pero el } 2 \text { l de junio ahime van a operar"(JI). }\end{array}$ \\
\hline & Relación con la madre & $\begin{array}{l}\text { "Yo creo que igual con mi mama siempre y conmigo } \\
\text { y con la doctora"(J2) }\end{array}$ \\
\hline & Relación con otros jóvenes & $\begin{array}{l}\text { "No es un tema de conversación. Pero yo conversé } \\
\text { con una niña. Ella ya terminó el tratamiento y dijo } \\
\text { que Dios le hizo un milagro. Yo ahi dije: Entonces Dios } \\
\text { me va a hacer un milagro a mí también. Ella dijo que } \\
\text { sufrió mucho si. Pero, ella salió de todo eso y ahora } \\
\text { viene solamente a hablar con la doctora.(JI) }\end{array}$ \\
\hline
\end{tabular}

Fuente: elaboración propia.

En cuanto al tercer objetivo, que era dar cuenta de las propuestas de aplicabilidad del asentimiento informado que realizan los distintos actores que participan del tratamiento de una patología oncológica en el Hospital Luís Calvo Mackenna, los resultados se expresan en el cuadro a continuación. 
Asentimientos informados en jóvenes de 12 a 17 años con patología oncológica: un dilema ético /López

Cuadro 8: Dimensión aplicabilidad de los asentimientos informados: equipo de salud.

\begin{tabular}{|c|c|c|}
\hline DIMENSION: & \multicolumn{2}{|l|}{ EQUIPO DE SALUD } \\
\hline Categorías Asociadas al Objetivo 3 & Sub Categorias & Relato del entrevistado \\
\hline \multirow[t]{5}{*}{ Implementación } & Requisitos & $\begin{array}{l}\text { "Principalmente evaluar la madurez del } \\
\text { paciente" (C12) }\end{array}$ \\
\hline & Relación equipo de salud/paciente & $\begin{array}{l}\text { "Crear una relación de confianza y dialogo } \\
\text { claro y abierto con el profesional encargado } \\
\text { de aplicarel asentimiento" (Cll) }\end{array}$ \\
\hline & Procedimientos & $\begin{array}{l}\text { "1. Evaluación psicológica, } 2 \text { reunión previa } \\
\text { con los padres, } 3 \text { conversación con el } \\
\text { equipo"(C9) }\end{array}$ \\
\hline & Tareas asignadas a padres/madres & $\begin{array}{l}\text { "La relación es muy importante ya que puede } \\
\text { empoderar o insegurizar al paciente"(Co) }\end{array}$ \\
\hline & Desafíos & $\begin{array}{l}\text { "...la aplicación como un proceso } \\
\text { continuo"(C2) }\end{array}$ \\
\hline
\end{tabular}

Fuente: elaboración propia.

Cuadro 9: Aplicabilidad de los asentimientos informados: madres cuidadoras

\begin{tabular}{l|l|}
\hline $\begin{array}{l}\text { DIMENSION: } \\
\text { APLICABILIDAD }\end{array}$ & CUIDADORAS (MADRES) \\
\hline Implementación & Requisitos \\
\hline & $\begin{array}{l}\text { "Podría ser que firmaran cuando son } \\
\text { quimios ambulatorias, en cosas que están } \\
\text { más relajadas para el niño"(M4) }\end{array}$ \\
\hline
\end{tabular}

Fuente: elaboración propia.

Cuadro 10: Aplicabilidad de los asentimientos informados: jóvenes pacientes oncológicos.

\begin{tabular}{l|l|l|}
\hline $\begin{array}{l}\text { DIMENSION: } \\
\text { Aplicabilidad }\end{array}$ & JÓVENES & \\
\hline Implementación & Procedimiento & $\begin{array}{l}\text { "Si me parecería firmar si la cosa no va } \\
\text { bien"(J2) }\end{array}$ \\
\hline
\end{tabular}

\section{Conclusiones}

Al momento de finalizar este estudio de caso, que buscó adentrase en el tema de los asentimientos informados en jóvenes de entre y 12 y 17 años, desde la mirada de tres actores involucrados en el 
proceso, como son los/las jóvenes, las cuidadoras (madres) y el equipo de salud, se formulan algunas conclusiones a partir de la matriz de discernimiento ético de análisis de contenido, a la que nos hemos referido en páginas anteriores.

Como ya se indicó, esta matriz plantea el desarrollo de un análisis que vincula los contenidos con tres mediaciones. Una primera mediación corresponde a la visión analítica que concierne al VER, la segunda mediación incumbe a la visión hermenéutica que se refiere al JUZGAR y la tercera mediación pertenece a la visión práctica que atañe al ACTUAR.

\section{Ver}

El asentimiento informado ha sido considerado como un derecho. Así ha ocurrido desde la mirada del equipo de salud, las cuidadoras y los/las jóvenes. Con mayor definición, este derecho ha sido dirigido hacia la información y la autonomía.

El equipo de salud ha aclarado que los/las jóvenes tienen derecho a recibir información, tal cual lo tiene cualquier otro ser humano. No es posible limitar este derecho a la edad de un/una joven, sino que lo que corresponde es que el lenguaje empleado por el equipo de salud sea capaz de cumplir con el objetivo de informar, de modo correcto y adecuándose al tiempo y a las características de las personas que son sus interlocutores. Así, cuando esta entrega de información se hace a un/una joven de entre 12 a 17 años, será el profesional el que deberá adecuarse a ello, para cumplir a cabalidad con este derecho.

Por su parte, las cuidadoras reconocen que este derecho a la información debe ser reconocido para sus hijos/hijas. Sin embargo, debe ser posible que los/las jóvenes puedan optar por ser o no informados. Este es un aspecto relevante, toda vez que se ve relacionado con la capacidad de autonomía, no teniendo que ver exclusivamente con no recibir información, sino, más bien, con la decisión de poder elegir el momento oportuno para ejercer este derecho. 
Tal como ha ocurrido con el resto de los actores claves, los/las jóvenes han relatado la importancia de validar su derecho a recibir información, porque es su propia vida la que se ve afectada. Los/ las jóvenes sienten que debe existir la posibilidad de conocer qué dirección es la que su vida lleva y no es posible la inexistencia de la comunicación para informar esto. Si bien la información puede ser entregada por sus padres, también manifiestan que sea el equipo de salud el que participe de este proceso.

Cuando la información es difícil para ellos porque las noticias que se aproximan no son las mejores, dicha información sigue siendo importante. En definitiva, la información de la enfermedad y sus temas asociados no pertenece al equipo de salud o a los cuidadores, sino que pertenece a quien se ve afectado por ésta, que es en definitiva quien la sufre.

Respecto del derecho a la autonomía, el equipo de salud ha señalado que este es un proceso que se debe facilitar en la atención de salud y para eso es necesario ir potenciando en el/la joven las habilidades para afrontar las dificultades en la sociedad actual.

Se requiere dar inicio a estas prácticas como parte de un proceso que va in crescendo. Así, también, este equipo relata que los/las jóvenes deben ser evaluados para demostrar la capacidad de autonomía, sin que esto sea asumido como un derecho.

En la autonomía, las cuidadoras han planteado que los/las jóvenes son "bastante niños" para tomar decisiones ante los tratamientos médicos y por ende, quienes deben decidir deben seguir siendo los padres ha ocurrido tal como histórica y legalmente. Se debiera entonces esperar hasta la cercanía de mayoría de edad para que ellos/ellas puedan tomar las decisiones en temas tan relevantes como su propia salud y lo que se hace con su cuerpo.

En los relatos de los/las jóvenes en relación con su autonomía, se tiende a mostrar que se desea regresar al hogar, buscando la protección y la seguridad, y encomiendan entonces la tarea de las decisiones a las madres, hasta el momento final del tratamiento. 
Sin perjuicio de lo anterior, los/las jóvenes han planteado que la toma de decisiones, no es una preocupación latente. Sin embargo, y con la misma claridad, han logrado realizar una definición crucial del momento en que sí se ven interesados y necesitan esgrimir su derecho a la autonomía y hacerlo valer.

El tiempo al que hacen referencia corresponde justamente al momento en que la vida se les va. Cuando el tratamiento tiene señales evidentes de fracaso; cuando no hay otras alternativas de tratamiento o cuando no hay otra posibilidad para ellos, los /las jóvenes claman su derecho a decidir. No puede haber un grito más potente que aquel de un ser humano que exige tomar decisiones sobre el fin de su vida.

Respecto de la categoría de adherencia, el equipo de salud ha planteado que cuanto mayor sea la información entregada a los/las jóvenes, pudiese verse favorecida considerablemente la adhesión al tratamiento $y$, por ende, las posibilidades de mejoría o estabilidad de la enfermedad.

El equipo de salud debiese ser el principal motor de la entrega de información a los/las jóvenes como una forma de aumentar este éxito en el tratamiento médico.

\section{Juzgar}

Las acciones para recuperar la salud deben estar centradas en la persona enferma y no en el equipo de salud. La dirección de la atención de salud debe iniciarse desde la persona que, en el contexto de la enfermedad, cuenta con mejores y mayores recursos emocionales y competencias para conducirlo. Quien está en esa posición debe ponerse al servicio de aquel que enfrentar el dolor de una enfermedad, por tanto, es el equipo de salud el llamado a acoger al ser humano que se vuelve débil y vulnerable ante esta situación.

Esto se vuelve todavía más crucial si quienes están enfermos son los/las jóvenes que están iniciando la vida y están aprendiendo a 
vivirla. Son los "adultos" quienes deben guiar amablemente este tránsito, desde el respeto, la justicia, la honestidad y la dignidad. En los tiempos actuales, que muestran un cierto grado de conciencia mayor acerca de los derechos de las personas y de cómo estas llegan a ejercerlos, sumado a la existencia de leyes que amparan estos derechos, no es posible que los equipos de salud se arroguen la decisión de informar o no. El derecho a ser informado debe ser considerado el más básico de los derechos en la atención de salud y el punto de partida de cualquier relación o vínculo que se dé entre los participantes de este proceso.

Parece ser que el aprendizaje social de control ha sido efectivo y los/las jóvenes han aprendido el mensaje de restarse de ejercer este derecho. Permanece latente el que los/las jóvenes no tienen derecho a la autonomía, a menos que sus habilidades para ello queden en evidencia.

Lo que se deja ver en este estudio, es que las atenciones de salud del hospital Luis Calvo Mackenna en la Unidad de Oncología se vinculan a los principios de una sociedad más bien conservadora, en la cual quienes toman decisiones son los adultos, los padres, es decir, los que "poseen los conocimientos".

El tema de los derechos resuena como un acto de buena voluntad, que proporciona la posibilidad de hacer las cosas de una forma diferente, pero que no alcanza para hablar de una conquista de derechos de los/las jóvenes. Asimismo, las madres cuidadoras han mostrado la misma visión que el equipo de salud, con tendencias más congruentes con un determinado ethos adultocéntrico. Claramente, asumir cambios sociales y culturales no es un proceso automático, sino por el contrario, de larga data. Debe cumplir, además, ciertos criterios de éxito que les permita a las personas confiar en que nuevos estilos de hacer las cosas también son fructíferos.

Pero, por otro lado, resulta también importante concluir de estos análisis de los relatos, que los/las jóvenes igualmente se adecuan a esta forma de proceder, volviéndose funcionales a la manera en que el equipo de salud y las madres cuidadoras enfrentan esta si- 
tuación. La realidad es que lo/las jóvenes no están preocupados y tampoco es de su interés estar preparados para validar por si mismos sus derechos en términos generales.

\section{Actuar}

De lo anterior se colige que el documento del asentimiento informado no resulta ser relevante ni para los/las jóvenes ni para las madres.

El equipo de salud, en tanto, que inicia este estudio declarando que el asentimiento no se aplica en la unidad, termina reconociendo que es un acto que debiese ser instalado en la misma Unidad de Oncología y también replicado en el resto del hospital. El equipo de salud, adicionalmente, considera que el asentimiento no solo habla de humanidad, sino que conjuntamente incluye principios de los que debe hacerse cargo, considerando que se trata de atenciones de salud.

Esto cobra importancia, toda vez que para que los/las jóvenes se encuentren más preparados para hacer valer sus derechos deben tener la posibilidad de practicarlos. Si se cuenta con este procedimiento, los/las jóvenes pensaran en su derecho y lo podrán poner en práctica, sin temores y con el respaldo de sus padres.

Esto coincide, ciertamente, con quienes deben conducir los actos en salud para poner la atención en los/las jóvenes que padecen una enfermedad. De existir este documento, se ofrecerán las oportunidades para que los/las jóvenes puedan tomarla. Este proceso será validado si es realizado de manera seria, respetuosa y responsablemente, y para eso se debe contar con horarios protegidos para su aplicación, trabajo interdisciplinario de profesiones de la salud y de las ciencias sociales, presencia de los padres y de las madres y cercanía con los pacientes.

Luego de este estudio y a las luz de los relatos, y por cierto de las conclusiones, surgen algunas inquietudes que se vinculan con los resultados reconocibles. 
Para este estudio no se hizo diferencia respecto de la procedencia territorial de las familias. Pudiese ser importante verificar si estos resultados serían los mismos entre familias de Santiago y de regiones, o si se presentarían diferencias en los relatos.

El factor económico no fue asumido en el estudio como una variable a considerar. Pudiese ser también que, existiendo diferencias en la situación económica, los relatos hubiesen sido distintos. Es posible plantearse preguntas como ¿qué ocurre con los/las jóvenes que no enfrentan enfermedades de esta naturaleza? ¿Será una variable relevante la enfermedad para que los relatos hayan asumido estas características o así será la forma en que los/las jóvenes responden ante el derecho a ser informados y a la toma de decisiones?

Sin duda, este estudio mostró lo que un tipo de juventud, tal como lo plantea Claudio Duarte, espera de su proceso de toma de decisiones ante una enfermedad, pero esto no es suficiente si no trasciende hacia cambios concretos que signifiquen la valoración de la opinión de los/las jóvenes. La invitación es a mirar y a escuchar a los/las jóvenes y proveer de un espacio contenido y protector para que, quienes quieran, puedan decidir cómo desean que sea el final de sus vidas.

\section{Bibliografía}

Amaro, M., Marrero, Á., Valencia, M., Casas, S. y Moynelo H. (1996). Principios Básicos de la Bioética. Revista Cubana de Enfermería, 12, 11-12. Recuperado el 23 de julio de 2017 de http:// scielo.sld.cu/scielo.php?script=sci_arttext\&pid=S086403191996000100006\&lng=es\&tlng=pt.

American Academy of Pediatrics. (1973). Model act providing for consent of minors for health services. Pediatrics [en línea], 1: 293. Recuperado de http://www.sccalp.org/boletin/193/ BolPediatr2005_45_156-160.pdf

Ander Egg, E. (1987). Técnicas de investigación social. Buenos Aires: Humanitas.

Arias, F. (2006). El proyecto de investigación: Introducción a la metodología científica. $5^{\text {a }}$ edición. Caracas-Venezuela: Episteme. 
Asenjo, J. (1995). Construcción dialógica de la personalidad moral. Revista Iberoamericana de Educación, 8.

Baeza, S. y Muñoz. M. (2012): Menores en Chile: ¿Cuándo son capaces o no son capaces? Recuperado de http://lyd.org/ centro-de-prensa/noticias/2012/02/menores-en-chile\%C2\%BFcuando-son-o-no-son-capaces

Beca, J. P. (2010). El Derecho al "Buen Morir". Recuperado el 26 de julio de 2015 de http://medicina.udd.cl/centro-bioetica/noticias/2010/08/05/el-derecho-al-buen-morir/.

Beloff, M. (1999) Modelo de protección integral. De los derechos del niño y de la situación irregular: Un modelo para armar y otro para desarmar. [En línea]. Revista Justicia y Derechos del niño, 1, 9-22. Recuperado el 11 de marzo de 2014 de http://www.unicef.org/argentina/spanish/ar_insumos_ PEJusticiayderechos1.pdf

Calderón, J. (2008) De la doctrina de la situación irregular a doctrina de la protección integral. Recuperado el 15 de mayo de 2016 de http://escribiendoderecho.blogspot.cl/2008/11/de-ladoctrina-de-la-situacin-irregular.html

Campos, G. y Lule, N. (2012) La observación, un método para el estudio de la realidad. Revista Xihmai, VII(13), 45-60.

Cillero, M. (1998). El interés superior del niño en el marco de la Convención Internacional sobre los Derechos del Niño. Justicia y Derechos del Niño, 125.

Delval, J. (1994). El desarrollo Humano. España: Siglo XXI.

Enesco, I. (2009). El concepto de la infancia a lo largo de la historia. [En línea]. Ensayos, Universidad Complutense de Madrid, 3.

Espinosa, C. V., Menoret, V., Puchulu, M. B., Selios, M.J. y Witriw, A. (2009). Bioética en la relación equipo de salud-paciente Revistas Diaeta, 27(128).

Fuenzalida, D. (2014). Protección jurídica y social de la infancia: situación actual en chile desde la perspectiva del derecho público. Santiago de Chile: Universidad de Chile, Facultad de Derecho, Departamento de Derecho Público.

Gamarra, G. (2015). Consentimiento Informado. Gaceta del Tribunal Nacional. Edt: Ética Médica.

Gilligan, C. (1985). La moral y la teoría. Psicología del desarrollo femenino. México: Fondo de Cultura Económica, p. 35.

Ginarte, Y. (2001). La adherencia terapéutica. Revista Cubana de Medicina General Integral, 17(5), 502-505. Recuperado el 27 de mayo de 2017, de http://scielo. 
sld.cu/scielo.php?script $=$ sci_arttext\&pid=S0864-

21252001000500016\&lng=es\&tlng=es.

González, P. (2007). Breve Reflexión sobre el consentimiento informado. Pediatría. Recuperado de http://www.cbioetica.org/revista/72/720410.pdf.

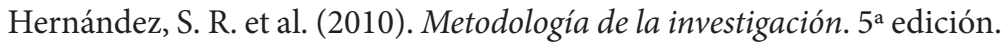
México: McGraw-Hill-Interamericana.

Iñiguez, L. (2003). Análisis del Discurso. Manual para las Ciencias Sociales. Barcelona: Editorial UOC, pp. 83-124.

Kohlberg, L. (1978). El niño como filósofo moral. Lecturas de Psicología del Niño, 2, 303-314.

Krause, M. (1995). La investigación cualitativa: un campo de posibilidades y desafíos. Revista Temas de Educación, 7, 19-39.

Ladriere, J. (1991). La ética en el Universo de la Racionalidad. Tucumán: UNSTA.

Lizana, R. (2010). Discurso de Trabajadores Sociales acerca de la Desigualdad Social. Una lectura ética. Tesis para optar al grado de Magíster en Ética Social y Desarrollo Humano, Facultad de Ciencias Sociales, Universidad Alberto Hurtado.

Martínez, C. (2013). Aspectos éticos en la adolescencia: del menor maduro al adulto autónomo. Revista Adolescere, I(2).

Makiya, M. (2013). Tratamiento de la Leucemia Linfoblástica Aguda Pediátrica, Recaída Hematológica, 17, 82-88.

Ministerio de Salud-Minsal. (2012). Carta deberes y Derechos.

Modelo de desarrollo económico de Navarra. Recuperado de https:// www.educacion.navarra.es/documents/27590/51352/ AUTONOMIA_Y_RESPONSABILIDAD.pdf

OMS. (2014). Evaluación del Sistema de Salud Mental en Chile. $2^{\circ}$ Informe, junio de 2014. Informe sobre la base del Instrumento de evaluación del sistema de salud mental de OMS (OMS IESM/WHO AIMS) Santiago de Chile.

ONU. (1990). Convención Internacional sobre los Derechos del Niño. Ginebra, Suiza.

Pineda, S. y Aliño, M. (2009). El concepto de Adolescencia. Manual de Practicas Clínica en la Adolescencia. La Habana, Cuba: MINSAP.

Pinto, B. J. y Gulfo, R. (2013). Asentimiento y consentimiento informado en pediatría: aspectos bioéticos y jurídicos en el contexto colombiano. Revista Colombiana de Bioética, 8(1), 144-165. 
Pyrrho, M., Cornelli, G. y Garrafa, V. (2009). Dignidad Humana: Reconocimiento y Operacionalización del Concepto. Acta Bioethica, 15(1), 65-69.

Puig, J. (1992). Criterios para educar moralmente en una sociedad democrática y plural. Comunicación, lenguaje y educación, $15,5-12$.

Quintero, J., Más, M. y Morera, L. (2007). El Consentimiento Informado en pacientes pediátricos con indicación de otocirugia por colostomía. Revista Habanera de Ciencia Médica, 6(1).

Rueda, L. (2008). Consentimiento informado en niños y adolescentes. Ars Medica, 37(1). Recuperado de http://www.arsmedica. cl/index.php/MED/article/view/126/63

Salvador Ortiz, M. (2004). Factores Psicológicos y Sociales Asociados a la Adherencia al Tratamiento en Adolescentes Diabéticos Tipo 1. Psykhe (Santiago), 13(1), 21-31.

Sánchez, A, (2009). El consentimiento informado y la relación médicopaciente. Revista de Bioética, 4-8. Recuperado de http:// www.cbioetica.org/revista/93/930407.pdf

Anderson, S. y Clarke, A. (2007). Manuel para Trabajadores de la Salud. Reino Unido: Child Friendly Healthcare Iniciative.

Urcola, M. (2003). Algunas apreciaciones sobre el concepto sociológico de juventud. INVENIO.

Vall, A. y Rodríguez, C. (2008). El derecho a la información del paciente: una aproximación legal y deontológica. BiD: textos universitaris de biblioteconomia i documentació, 21 (desembre). DOI: http://dx.doi.org/10.1344/105.000000324

Valles, M. (1999). Técnicas Cualitativas de la Investigación Social. Madrid: Sintesis Sociología.

Zerpa, C. (2007). Tres teorías del desarrollo del juicio moral: Kohlberg, Rest, Lind. Implicaciones para la formación moral. Laurus, 13(23), 137-157. 